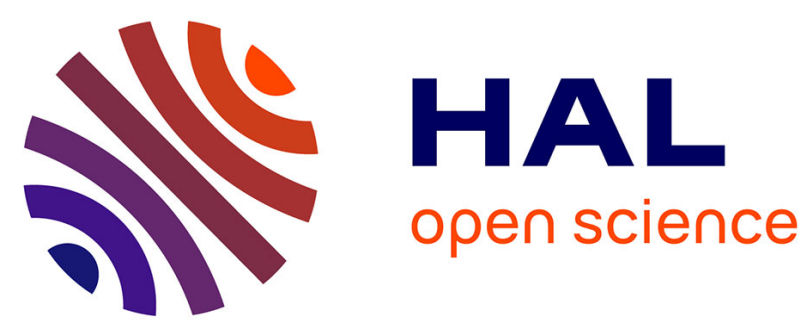

\title{
A performance analysis of distributed QoS negociation during establishment session
}

\author{
Anis Zouari, Karine Guillouard, Jean-Marie Bonnin
}

\section{To cite this version:}

Anis Zouari, Karine Guillouard, Jean-Marie Bonnin. A performance analysis of distributed QoS negociation during establishment session. Q2SWinet '07: Proceedings of the 3rd ACM workshop on QoS and security for wireless and mobile networks, Oct 2007, Chania, Crete Island, Greece. pp.160 $163,10.1145 / 1298239.1298269$. hal-02900907

\section{HAL Id: hal-02900907 https://hal.science/hal-02900907}

Submitted on 16 Jul 2020

HAL is a multi-disciplinary open access archive for the deposit and dissemination of scientific research documents, whether they are published or not. The documents may come from teaching and research institutions in France or abroad, or from public or private research centers.
L'archive ouverte pluridisciplinaire HAL, est destinée au dépôt et à la diffusion de documents scientifiques de niveau recherche, publiés ou non, émanant des établissements d'enseignement et de recherche français ou étrangers, des laboratoires publics ou privés. 


\section{A Performance Analysis of Distributed QoS Negotiation During Establishment Session}

\author{
Anis Zouari \\ France Telecom \\ 4, Rue du Clos Courtel \\ Rennes, France \\ anis.zouari2@orangeftgro \\ up.com
}

\author{
Karine Guillouard \\ France Telecom \\ 4, Rue du Clos Courtel \\ Rennes, France \\ karine.guillouard@orangef \\ tgroup.com
}

\author{
Jean-Marie Bonnin \\ ENST Bretagne \\ 2, Rue de la Châtaigneraie \\ Rennes, France \\ jm.bonnin@enstbretagne.fr
}

\begin{abstract}
In order to achieve guaranteed services, we need to negotiate network resources providing the required quality of services (QoS). For this reason, the common set-up process of service session is divided into three phases. At first, the application specifies its QoS requirements, then, the QoS parameters at IP level and MAC level are successively specified and finally the network applies the negotiated QoS configuration. In this paper, we propose to negotiate at the same time the QoS parameters at both levels. An optimization of this approach has also been discussed. This optimization focuses on distributing negotiated QoS parameters between several entities located in different hierarchical levels. The assignment of parameters is dynamically configured. Therefore, this paper proposes a performance model to compare the new combined-distributed negotiation process with the negotiation processes applied in UMTS Release 5 (R5) [4] during session establishment. Numerical results show that the proposed approach is more scalable on the network side than the UMTS approach.
\end{abstract}

\section{Categories and Subject Descriptors}

H.4.3 [Information System Application]: Communication Applications.

\section{General Terms}

Management, Performance, Design.

\section{Keywords}

QoS negotiation, session establishment, mobility.

\section{INTRODUCTION}

Providing end to end QoS over Internet can be divided into three successive phases: negotiation, decision and reservation. At the negotiation phase, QoS parameters needed by the application in use are specified. Then, these negotiated parameters are checked in order to validate their values. Finally, the requesting entity will receive an acknowledgement with a set of parameters acceptable

Permission to make digital or hard copies of all or part of this work for personal or classroom use is granted without fee provided that copies are not made or distributed for profit or commercial advantage and that copies bear this notice and the full citation on the first page. To copy otherwise, or republish, to post on servers or to redistribute to lists, requires prior specific permission and/or a fee.

Q2SWinet'07, October 22, 2007, Chania, Crete Island, Greece.

Copyright 2007 ACM 978-1-59593-806-0/07/0010_..\$5.00. along the path. In heterogeneous environment, we should provide QoS in both IP and radio access networks (IPAN and RAN). For this purpose, two approaches are defined. They are differentiated by the chronological order of resource negotiations. For instance, in UMTS R5 [4], IP QoS parameters are negotiated at first and then the negotiation of radio resources is started. Unlike UMTS R5, DAIDALOS IST project [2] starts by providing QoS on RANs and then on IPANs.

It should be noted that QoS solutions, based on these approaches, are characterized by a restricted communication between IP and link layers. For instance, in the approach adopted in UMTS R5, IP resources are negotiated without tacking into account current radio resources. As a result, for negotiated IP resources, available link layer resources may not be enough to satisfy application QoS requirements. In addition, both approaches separate the negotiation process on RAN side from the negotiation process on IPAN side. As a result, the amount of exchanged QoS signalling data may increase significantly in heterogeneous environments. For these reasons, we propose to negotiate IP resources and its correspondent radio resources. Our work focuses on integrating this approach in the solution proposed in [5]. In [5], authors present an approach for distributing the handover (HO) decision function over multiple entities within networks and end terminals. Therefore, this approach allows the construction of a handover decision chain specifying entities which are able to control $\mathrm{HO}$ decision. This chain is constructed in a hierarchical and distributed manner. The initial results, given in [5], drive our work to test the use of this approach in the negotiation phase. For this reason, we have defined a QoS negotiation chain. This chain includes the list of entities able to negotiate QoS parameters. It also specifies the list of QoS parameters allocated to each entity participating in QoS negotiation.

This paper is structured as follows. Section 2 presents an example of QoS negotiation scenario. This scenario is applied in UMTS R5 during a session establishment. Then, section 3 presents the scenario of session establishment implemented in a new proposed Hierarchical-Distributed architecture. Section 4 introduces the results of theoretical study on resource negotiation performance. Finally, concluding remarks are given in section 5.

\section{UMTS R5 SESSION ESTABLISHMENT}

IP Multimedia Subsystem (IMS) [4] is the architecture chosen by the $3 \mathrm{GPP}$ consortium for UMTS R5 and later. IMS is the answer to actual trend, which is to shift data and multimedia services to Packet Switched (PS) domain. Figure 1shows an overview of one 
possible QoS negotiation scenario which is adopted in UMTS R5. This scenario is divided into the following steps $(\mathrm{S})$.

- Specifying Application Requirements (S1): At session set-up, application layer between the end hosts identifies the QoS requirements. Then, these application layer QoS requirements are communicated via the Session Initiation Protocol (SIP) [1]. In order to provide QoS in IP access network, a context is established as specified in the Packet Data Protocol (PDP).

- Triggering and Establishing QoS Negotiation at IP Level (S $2+3$ ): On receiving the acknowledgement message of the SIP session set-up, end terminal establishes the suitable PDP context and activates it by sending an "Activate PDP context" message to SGSN with the UMTS QoS parameters. Then, SGSN sends a "Create PDP Context" message to GGSN. On receiving this message, GGSN checks if there are enough resources to provide QoS requirements. In addition, it checks if terminal is authorized to use the required resources. Finally, GGSN decides either to validate or to refuse the QoS request and sends a "Create PDP Context Response" message back to SGSN.

- Triggering and establishing QoS negotiation at radio level (S 4+5): On receiving GGSN response indicating that QoS request has been accepted, SGSN sends "RAB Assignment Request" message to candidate RNCs. Each RNC checks if there are enough radio resources to satisfy the QoS request. Then, it sends to SGSN the result of its analysis in "RAB Assignment Response" message.

- Ending QoS negotiation (S 6): SGSN sends "Activate PDP Context Accept" message to end terminal. This message contains the specification of negotiated QoS parameters to be used during this session.

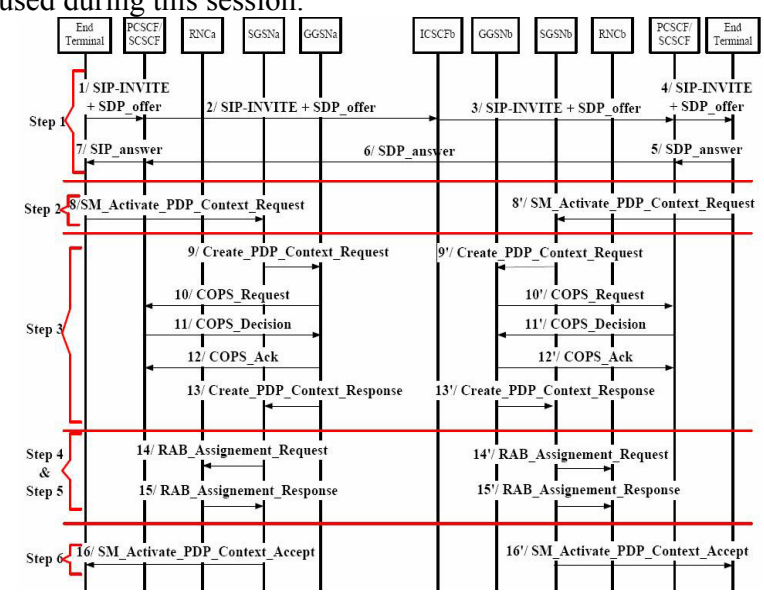

Figure 1: Resource negotiation in case of UMTS R5

Since radio level is considered as more constringent than IP level, it is very probable that the response from the link level negotiation indicates a QoS different from the one that is possibly negotiated at the IP level. Consequently, in UMTS, QoS renegotiation may be required at IP level. Accordingly it may be useful to perform a negotiation at link level then at IP level. This has been implemented in the case of DAIDALOS. In DAIDALOS, at first, radio admission control is performed in order to allocate differentiated service code to the application traffic. Then, all this information is sent to all candidate ARs to apply IP resource negotiation and reservation. In the following section, a new proposed approach is presented. This approach defines several distributed and common entities to deal simultaneously with IP and radio access network negotiations. This approach seems to be more efficient and scalable than the two other approaches.

\section{THE PROPOSED APPROACH}

The current trends look for spreading the use of mobile systems in heterogeneous environment. For instance, nowadays, end terminal could be connected to three different access networks: WiMAX, WLAN and UMTS. UMTS and WLAN connections are provided by the same operator X while WiMAX connection is provided by the operator Y. In this example, three hierarchical levels of management can be distinguished: end terminal remains connected to the same RAN but it changes its point of attachment (PoA), end terminal changes its RAN but it remains associated to the same operator and end terminal changes either its operator or its IPAN. Supporting hierarchical managements implies the use of a hierarchical architecture approach in the new proposed solutions. In addition, as depicted in [3], centralized architecture is likely to encounter scalability and reliability problems compared to distributed architecture. For these reasons, we think that an architecture supporting our negotiation approach, should be at the same time a distributed and hierarchical architecture. This section is composed of two parts. In the first part, the three managers implemented in our proposed architecture, namely Hierarchical and Distributed (HD) architecture, are presented. These managers are: radio manager (RM) allocated to each radio access network, IP access network manager (IPAM) allocated to each IP access network and operator manager (OM) allocated to each operator domain. The second part gives an example of session establishment scenario applied in HD architecture.

\subsection{Managers Defined in HD Architecture}

The proposed HD architecture defines the following managers:

- Operator manager (OM): It manages customer session and operator preferences related to the choice of network for a specific user. It is also responsible for managing inter-operator and inter IP access network HOs. Therefore, it can trigger a handover to apply the operator requirements.

- IP access network manager (IPAM): It triggers and manages inter-radio access network HO where old and new RANs should be associated to the same IP access network. In addition, IPAMs located in the same operator area could establish QoS context transfer or QoS requirement negotiation.

- Radio access network manager (RM): It may receive fine radio measurements from the terminal regarding the neighbouring radio access points belonging to same access technology. Therefore, it includes some further information such as a list of candidate RANs to which an end terminal is connected or can establish a connection. QoS context can be transferred directly between RMs. Moreover, RM is able to participate in network controlled or assisted QoS negotiation.

\subsection{Session Establishment in HD Architecture}

In HD architecture, QoS parameters are shared between three hierarchical managers: OM at operator core network level, IPAMs at IP access network and RMs located in each RAN within a domain. In other words, a list of several QoS parameters is allocated to each manager. During AC and resource reservations, 
each manager is responsible for negotiating its allocated list of parameters, taking a partial decision (i.e. manager decides to admit or to refuse application traffic according to its allocated parameters) and sending its partial decision to lower node (e.g. another manager or terminal). QoS reservations are then performed by the execution module at PoA and terminal. Figure 2 illustrates a simplified example of session initiation using SIP as signalling protocol. This scenario is divided into three steps:

- Specifying application requirements (step 1): In order to start the session caller terminal sends "INVITE" message containing QoS offer to its OMa. OMa forwards this message to called terminal via its $\mathrm{OMb}$. Called terminal analyzes the received offer and sends its response in "SDP answer" message. In this scenario, let's suppose that the proposed offer is accepted.

- Combining negotiation (step 2): OMa intercepts "SDP answer" message in order to check if its allocated QoS parameters (e.g. connection cost, security requirements) satisfy the requirements included in the QoS offer. As a result, the operator manager (OMa) takes a partial decision based only on its allocated QoS parameters. Then, it sends this decision in the "HDHO Needed Ind" message to IPAMa. On receiving this message, IPAMa collects IP parameters. These parameters are used for the simultaneous negotiation. In fact, they are used locally to add some new information about parameters stored in IPAM (e.g. transmission delay) to the received partial decision. IP parameters are also sent to lower nodes in the "HDHO Needed Ind" message in order to be compared with several radio parameters. Thus, on receiving this message, RMa checks its allocated radio parameters (e.g. network load), completes the received partial decision and sends it to the caller terminal.

- Ending QoS negotiation (step 3): Finally, end terminal takes the final decision (i.e. it chooses a PoA to be connected to) and then starts resource reservation process (e.g. packet data protocol). These messages are also used to inform all entities on the end to end path about the final decision.

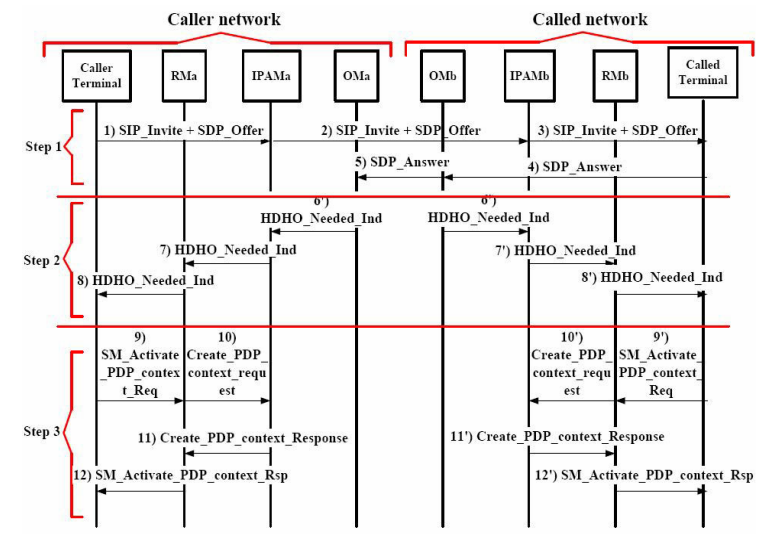

Figure 2: Resource negotiation in case of $\mathrm{HD}$ architecture

\section{PERFORMANCE ANALYSIS OF QOS NEGOTIATION}

This section gives a performance model to study the cost of QoS negotiation process in term of consumed bandwidth according to the aforementioned scenarios. This study is based on messages exchanged in these scenarios. In addition, this study assumes that messages exchanged during QoS negotiation use User Datagram Protocol (UDP) at transport layer. Consequently, these messages must be contained in one UDP/IP packet. Each message is mainly composed of two parts: protocol headers namely overhead and data. As previously stated, QoS signalling message travels over an UDP segment which is encapsulated in an IP packet. For this reason, the total overhead, namely $\mathrm{O}$, needed to send a QoS signalling message is the sum of the overhead introduced by UDP and IP protocols (i.e. $\mathrm{O}=56$ bytes).

The second part of QoS signalling message contains some needed QoS information (e.g. applications requirements, required or needed values of some QoS parameters, etc.). In the following sections, $\mathrm{S}_{\mathrm{QoSd}}$ refers to the maximum size of QoS data in a message. $\mathrm{S}_{\mathrm{OM}}$ is the size of QoS data sent from OM to IPAM. $\mathrm{S}_{\text {IPAM }}$ is the size of QoS data sent from IPAM to RM.

$\mathrm{S}_{\mathrm{RM}}$ is the size of QoS data sent from RM to end terminal. It has to be noted that $\mathrm{S}_{\mathrm{Q} O S d}=\mathrm{S}_{\mathrm{OM}}+\mathrm{S}_{\mathrm{RM}}+\mathrm{S}_{\mathrm{IPAM}}$. Finally, 1 represents respectively the number of RNC for UMTS R5 scenario and the number of RM for HD architecture scenario. We assume that all RNCs are associated to only one SGSN and GGSN and all RMs are attached to the same IPAM managed by one OM.

$B_{\text {scenario-participant }}$ is a notation used to refer the upper bounds on the total amount of data sent/received by participant according to the studied scenario, where participant could be Terminal, SGSN, GGSN, RNC, OM, IPAM, RM or Network and scenario $\in\left\{\mathrm{UMTS}_{\mathrm{R} 5}, \mathrm{HD}\right\}$. In this study, the formulation of $\mathrm{B}_{\text {scenario-participant }}$ for the different studied cases is given by the following equations.

$$
\begin{aligned}
& B_{U M T S_{-}}^{T e r m i n a l}=4 * S_{Q o S d}+184 \\
& B_{U M T S_{-} R 5}^{G G S N_{1}}=12 * S_{Q o S d}+552 \\
& B_{U M T S_{-}}^{S G S}=2 *(l+2) *\left(S_{Q o S d}+46\right) \\
& B_{U M T S_{-} R 5}^{R N C}=2 * S_{Q o S d}+92 \\
& B_{U M T S_{-}}^{\text {Netwo }}=(11+2 * l) * S_{Q o S d}+874 \\
& B_{H D}^{T e r m i n a l}=3 * S_{Q o S d}+l * S_{R M}+(l+3) * 46 \\
& B_{H D}^{O M}=3 * S_{Q o S d}+S_{O M}+184 \\
& B_{H D}^{I P A M}=3 * S_{Q o S d}+S_{O M}+l *\left(S_{R M}\right. \\
& \left.+S_{I P A M}\right)+(l+4) * 46 \\
& B_{H D}^{R M}=4 * S_{Q o S d}+2 * S_{R M}+S_{I P A M}+276 \\
& B_{H D}^{N \text { etwork }}=8 * S_{Q o S d}+S_{O M}+l * S_{I P A M} \\
& +2 * S_{R M}+(2 * l+9) * 46
\end{aligned}
$$

We notice that the amount of data sent/received by each participant in HD architecture scenario given by equations (11), (12), (13), (14) and (15) are a function of at least two variables (i.e. $\mathrm{S}_{\mathrm{OM}}, \mathrm{S}_{\mathrm{RM}}, \mathrm{S}_{\mathrm{IPAM}}$ or $\mathrm{S}_{\mathrm{QoSd}}$ ). In order to study the three scenarios, all equations should be given as a function of the same variable. In this study, the chosen common variable is $\mathrm{S}_{\mathrm{QoSd}}$. Consequently, $\mathrm{S}_{\mathrm{OM}}, \mathrm{S}_{\mathrm{RM}}$ and $\mathrm{S}_{\mathrm{IPAM}}$ should be given as a function of $\mathrm{S}_{\mathrm{QoSd}}$. For this reason, we have defined four possible distributions of QoS data over different participating entities in the HD architecture scenario (i.e. OM, RM and IPAM). In the first, noted HD scenario 1, we suppose that QoS data are equally shared between the different entities (i.e. $\mathrm{SOM}=\mathrm{S}_{\mathrm{RM}}=\mathrm{S}_{\mathrm{IPAM}}=$ $\left.1 / 3 \mathrm{~S}_{\mathrm{QoSd}}\right)$. In the second, termed HD scenario 2, we suppose that the most of data are stored in IPAM (i.e. $\mathrm{S}_{\mathrm{OM}}=\mathrm{S}_{\mathrm{RM}}=1 / 6 \mathrm{~S}_{\mathrm{IPAM}}=$ $\left.1 / 8 \mathrm{~S}_{\mathrm{QoSd}}\right)$. In the third, namely HD scenario 3 , we suppose that the most of QoS data are stored in RM (i.e. $\mathrm{S}_{\mathrm{OM}}=1 / 6 \mathrm{~S}_{-}\{\mathrm{RM}\}=$ 
$\left.\mathrm{S}_{\mathrm{IPAM}}=1 / 8 \mathrm{~S}_{\mathrm{QoSd}}\right)$. In the last case, we suppose that the most of QoS data are stored in $\mathrm{OM}$ (i.e. $\mathrm{S}_{\mathrm{RM}}=1 / 6 \mathrm{~S}_{\mathrm{OM}}=\mathrm{S}_{\mathrm{IPAM}}=1 / 8$ $\left.\mathrm{S}_{\mathrm{QoSd}}\right)$.

\subsection{Data Exchanged on End Terminal Side}

As depicted in Figure 3 a), the amount of QoS data exchanged in HD architecture is dependent on QoS data size stored in each manager. For instance, if most QoS parameters are allocated to manager in radio access network, end terminal would be the most loaded. Therefore, less QoS parameters are stored in radio manager (RM), less end terminal is loaded. This is due to the communication established between terminal and RM in order to receive QoS information about the proposed QoS from each candidate RM (i.e. RM partial data). In UMTS, it is the SGSN which receives this information from each candidate RNC. In order to reduce end terminal load in HD architecture we can propose the use of network controlled session establishment.

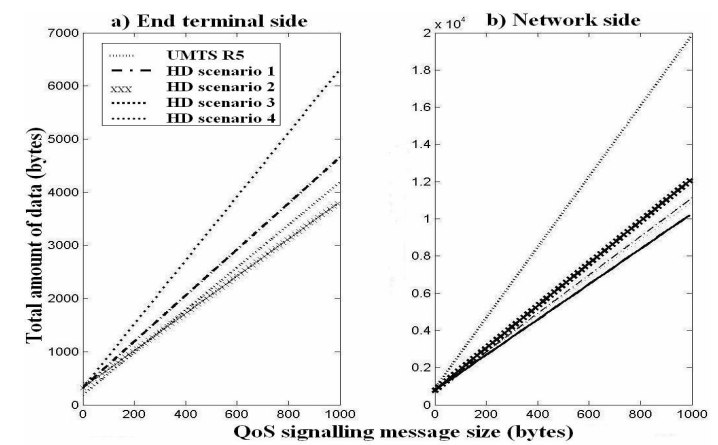

Figure 3: Data exchanged on terminal and network sides

\subsection{Data Exchanged on Network Side}

The evolution of the amount of data received/sent by all entities located in the network is depicted in Figure $3 \mathrm{~b}$ ). We notice that network in case of HD architecture is less loaded than in UMTS scenario. In fact, this is mainly due to the assignment of a list of QoS parameters to each manager (i.e. OM, RM and IPAM). For instance, IPAM did no longer send all QoS parameter requirements but only a list of pre-assigned required parameters. Network load in HD architecture does not vary a lot when the amount of QoS data allocated to each manager is changed.

\subsection{Comparing Data Exchanged in Managers}

Unlike RNC, radio manager, in HD architecture, is able to communicate directly with end terminal. Thus, it implements more functionalities than RNC in UMTS architecture. For this reason, as depicted in Figure $4 \mathrm{c}$ ), data exchanged by radio manager is more important than data exchanged by RNC. The result of sharing QoS parameters between several entities is brought out on comparing data exchanged in SGSN and IPAM, as depicted in Figure 4 a), and also on comparing data exchanged in GGSN and OM, as depicted in Figure 4 b). In fact, IPAM and OM are less loaded than SGSN and GGSN in UMTS scenario

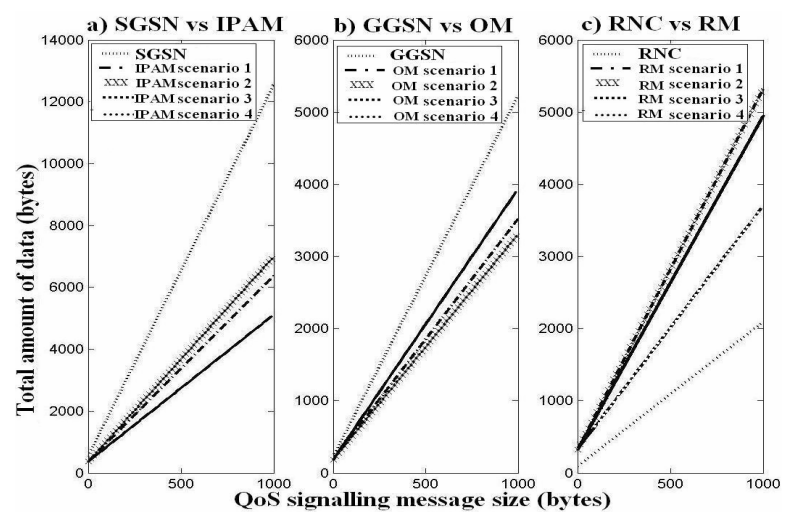

Figure 4: Data exchanged in RM, IPAM and OM

\section{CONCLUSION}

This paper proposed to combine negotiation at IP and radio levels during session establishment. Therefore, an optimization is introduced to this approach. This optimization makes use of the fact that QoS parameters are collected from several entities located in different hierarchical levels. This information can be used locally during QoS negotiation and thereby reduce the amount of QoS data included in QoS signalling messages. A performance analysis of distributing QoS parameters over multiple entities able to communicate together has been performed in this paper. In this study, we compared the amount of data exchanged in two scenarios differentiated on the basis of the negotiation approach in use. Our analysis points out that the HD architecture seems to be an alternative operator-centric solution to improve QoS management on network side. In fact, data exchanged on network side in HD architecture scenario is less than in UMTS scenario. However, this solution increases the amount of data exchanged on end terminal side. This amount can be reduced by adopting network assisted or controlled QoS management approaches. Noticed that this optimization can also be applied during handover management. We are currently assessing the performance of our optimization in such a case.

\section{REFERENCES}

[1] J. R. et al. Sip: Session initiation protocol. IETF RFC 3261, June 2002.

[2] R. L. A. et al. Scalable QoS aware mobility for future mobile operators. IEEE communication Magazine, June 2006.

[3] S. R. Lima, P. Carvalho, and V. Freitasl. Admission control in Multiservice IP networks: Architectural Issues and Trends. IEEE Communications Magazine, April 2007.

[4] T. S. G. Services and S. Aspects. IP Multimedia Subsystem (IMS) (Release 5). 3GPP TS 23.228, December 2004.

[5] L. Suciu and K. Guillouard. A hierarchical and distributed handover management approach for heterogeneous networking environments. The 3 International Conference on Networking and Services, Athens, Greece, June 2007. 